\title{
BINDING TO A LIPID LAYER: A LOW MATERIAL CONSUMING APPROACH FOR STRUCTURAL ANALYSIS OF MEMBRANE PROTEINS.
}

Lévy, D.

Institut Curie, Section de Recherche, UMR CNRS 168, 75231 Paris, France

Difficulties of over-expression of membrane proteins represent a bottleneck for their structural analysis. We have developed strategies based on the specific binding of membrane proteins to a lipid layer spread at the air/water interface, which allowed decreasing to 1-2 microgram the amount of proteins per structural experiment. A first approach is the production of $2 \mathrm{D}$ crystals that can be analysed by electron crystallography. It involves a binding step of the micellar proteins to the lipid layer, followed by reconstitution of the proteins into a lipid bilayer ${ }^{1}$ (Fig. 1). $2 \mathrm{D}$ crystals of different his-tagged proteins have been obtained after specific binding to a Nichelating lipid layer, and of charged proteins after electrostatic binding to a charged lipid layer. Since the fist 2D crystals obtained in 1999, up to 13 different membrane proteins have been crystallized by the lipid layer approach by us or other groups revealing the general use of this approach. A second strategy consists to bind and to orient single proteins onto the lipid layer. The unique orientation of bound proteins provided rapidly an averaged projection useful per se or a missing projection crucial for a 3D reconstruction by single particles analysis. This has been successfully used in the case of $\mathrm{YvcC}$, a multidrug transporter from Bacillus subtilis, member of the ATP-binding cassette superfamily, highly homologous to each half of human multidrugresistance P-glycoprotein and to several other bacterial half-ABC transporters. Controlled and partial detergent removal from YvcC-lipid micelles allowed the production of particularly interesting lipid-detergent-YvcC ring-shaped particles, about $40 \mathrm{~nm}$ in diameter, well suited for single particle analysis. Analysis reveals a preferred orientation of the particle onto the grid leading to a reconstruction with a large missing cone. Binding of these histidine-tagged ringshaped particles to lipid layers functionalized with a $\mathrm{Ni}^{2+}$-chelating head group generated a preferential perpendicular orientation, eliminating the missing cone in the final three-dimensional reconstruction. From such analysis, a computed volume has been determined to $2.5 \mathrm{~nm}$ resolution giving a detailed insight into the structural organization of this half- $\mathrm{ABC}$ transporter within a membrane. (fig. 2)

Lévy, D., Chami, M. and Rigaud J.L. (2001) Two-dimensional crystallization of membrane proteins: the lipid layer strategy. FEBS Lett. Review . 504,187-93.

Chami, M., Steinfels, E., Orelle, C., Jault, JM., Di Pietro, A., Rigaud, JL. And Marco, S. (2002)

Three-dimensional structure by cryo-electron microscopy of YvcC, an homodimeric ATPbinding cassette transporter from Bacillus subtilis. J. Mol. Biol. 315, 1075-1085. 
Fig. 1: 2D crystallization of membrane protein by the lipid layer methods. a) binding of micella lipid/protein/detergent micelles to a functionalised lipid layer at the air/water interface. $\mathrm{b}$ Reconstitution of proteins into a lipid bilayer upon detergent removal. c) 2D crystallization.

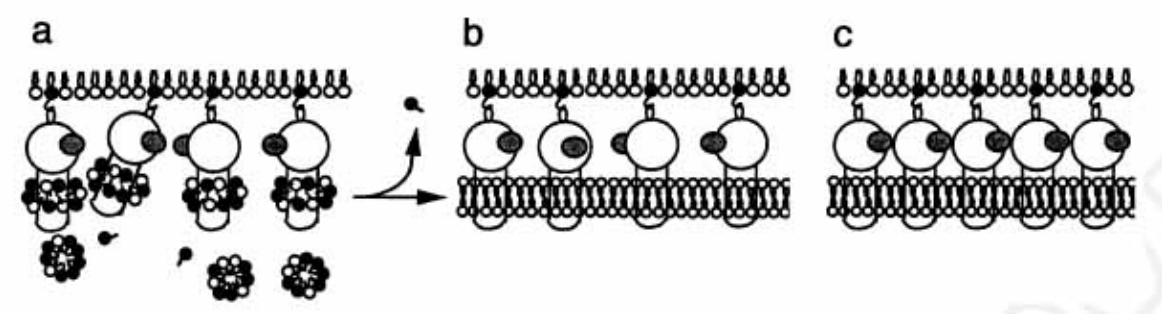

Figure $23 \mathrm{D}$ reconstruction of the bacterial ABC transporter $\mathrm{YvcC}$ at $2.5 \mathrm{~nm}$ resolution by cryoEM. A) ring-like structures of 24 homodimers obtained by partial detergent removal. The average of particles spread onto a holey grid reveals mainly top views. B) side views obtained by binding onto an Ni-NTA lipid layer. C) 3D reconstruction. D) NBD domains. E) transmembran domains

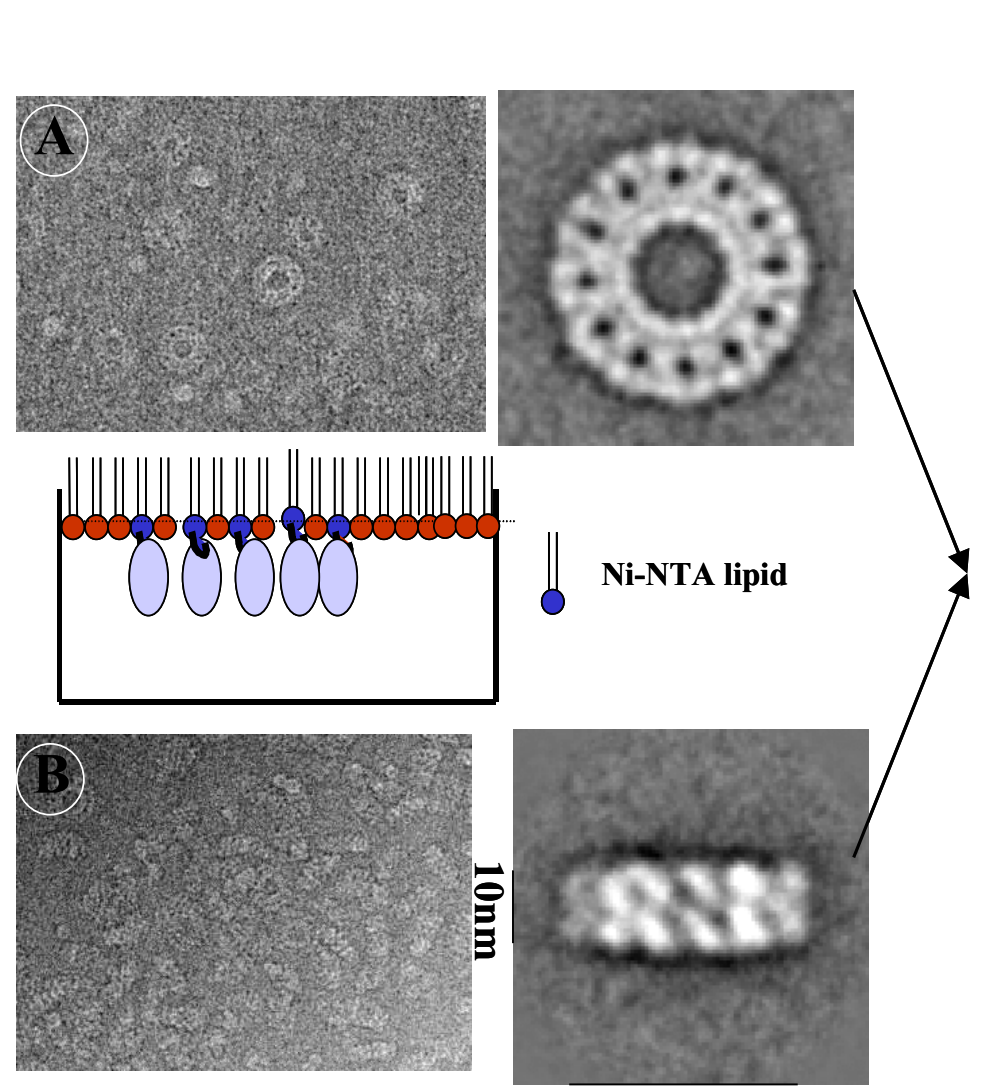

40nm

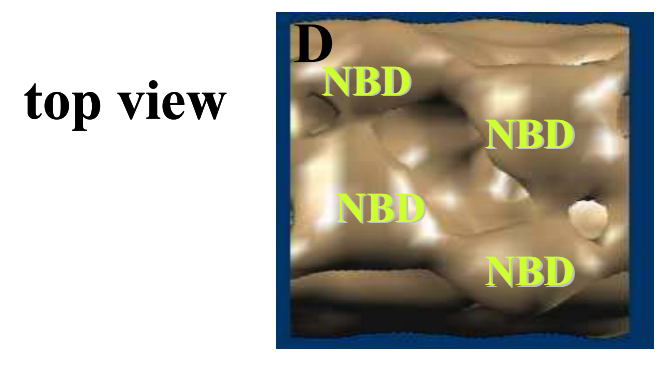

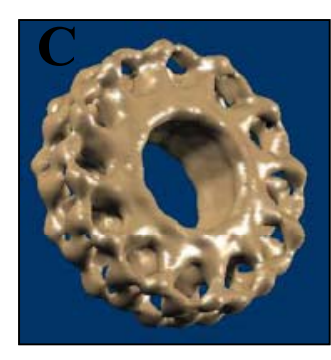

side view

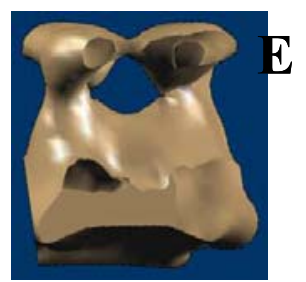

YvcC Homodimer 